\title{
NUMERICAL INVESTIGATION OF HEAT TRANSFER IN A TURBULENT FLOW IN CHANNELS WITH GAP
}

\begin{abstract}
S. I. S. Souza a ,
H. A. Martins ${ }^{\mathrm{a}}$, and J. N. V. Goulart ${ }^{\mathrm{b}}$

${ }^{a}$ Universidade Federal do Rio Grande do Norte Departamento de Engenharia Mecânica CEP 59072-970, Natal, RN, Brasil sandi@ufrn.br

${ }^{\mathrm{b}}$ Universidade de Brasília Campus Gama, Área Especial de Indústria 1 CEP 72444-210, Gama, DF, Brasil.
\end{abstract} Received: October 29, 2014 Revised: November 30, 2014 Accepted: December 30, 2014

Keywords: turbulent flow, compound channels, URANS, channel with slot, large scale structures

\section{NOMENCLATURE}

$\mathrm{C}_{\mathrm{f}} \quad$ mean skin friction coefficient

d slot length, $m$

D diameter, $\mathrm{m}$

f frequency, $\mathrm{s}^{-1}$

$\mathrm{F}_{1}, \mathrm{~F}_{2}$ blending functions

g slot thickness, $\mathrm{m}$

$\mathrm{h}$ enthalpy, convective heat transfer coefficient $\mathrm{J} / \mathrm{kg}, \mathrm{W} / \mathrm{m}^{2} \mathrm{~K}$

$\mathrm{h}_{\text {tot }} \quad$ total enthalpy, $\mathrm{J} / \mathrm{kg}$

$\mathrm{k}$ turbulent kinetic energy, $\mathrm{m}^{2} / \mathrm{s}^{-2}$

$\mathrm{L}$ length, length scale, $\mathrm{m}$

$\mathrm{L}_{\mathrm{vk}} \quad$ Von Karman length scale

$\mathrm{Nu}$ Nusselt number

$\mathrm{p}$ pressure, $\mathrm{Pa}$

$\mathrm{P}_{\mathrm{k}} \quad$ turbulence production, $\mathrm{kg} / \mathrm{m} \mathrm{s}^{3}$

$\mathrm{Pr}_{t} \quad$ turbulent Prandtl number

q" heat flux, W/m ${ }^{2}$

QSAS source term of the SAS turbulence model, $\mathrm{W} / \mathrm{m}^{3}$

$\mathrm{S}_{\mathrm{E}} \quad$ source term to Reynolds average energy conservation equation, $\mathrm{kg} / \mathrm{m} \mathrm{s}^{3}$

$\mathrm{S}_{\mathrm{M}} \quad$ body forces, $\mathrm{kg} / \mathrm{m}^{2} \mathrm{~s}^{2}$

St Strouhal number

$\mathrm{t}$ time, $\mathrm{s}$

$\mathrm{T}$ temperature, ${ }^{\circ} \mathrm{C}$

$\overline{\mathrm{T}}_{\mathrm{f}} \quad$ local mean temperature of the fluid, ${ }^{\circ} \mathrm{C}$

$\overline{\mathrm{T}}_{\mathrm{r}} \quad$ local mean temperature of the wall tube, ${ }^{\circ} \mathrm{C}$

$\mathrm{U} \quad$ velocity vector, $\mathrm{m} / \mathrm{s}$

$\mathrm{U}_{1}$ axial velocity, $\mathrm{m} / \mathrm{s}$

$\mathrm{U}_{\mathrm{c}}$ convection velocity, $\mathrm{m} / \mathrm{s}$

$\mathrm{U}_{\mathrm{ed}}$ edge velocity, $\mathrm{m} / \mathrm{s}$
$\mathrm{V}_{\text {bulk }}$ bulk velocity, $\mathrm{m} / \mathrm{s}$

W transversal velocity, span-wise velocity, $\mathrm{m} / \mathrm{s}$

$\mathrm{X}$ axial distance, $\mathrm{m}$

$\mathrm{X}$ coordinate

$\mathrm{Y}^{+}$dimensionless distance between the wall the closest point

$\mathrm{Z}$ transversal distance, $\mathrm{m}$

\section{Greek symbols}

$\rho$ density, $\mathrm{kg} / \mathrm{m}^{3}$

$\delta \quad$ delta of kronecker

$\Delta \mathrm{x}$ distance, $\mathrm{m}$

$\theta \quad$ Angular position, ${ }^{\circ}$

$\lambda$ thermal conductivity, $\mathrm{W} / \mathrm{m} \mathrm{K}$

$\lambda_{1}$ streamwise spacing between alternate sequence of large structures, $\mathrm{m}$

$\mu \quad$ dynamic vicosity, $\mathrm{kg} / \mathrm{m} \mathrm{s}$

$\mu_{\mathrm{t}} \quad$ eddy viscosity, $\mathrm{kg} / \mathrm{m} \mathrm{s}$

$\tau$ molecular tensor, $\mathrm{kg} / \mathrm{m} \mathrm{s}^{2}$

$\tau_{\mathrm{w}} \quad$ wall shear stress, $\mathrm{kg} / \mathrm{m} \mathrm{s}^{2}$

$\omega$ specific dissipation rate, $\mathrm{m}^{2} / \mathrm{s}^{3}$

$\Omega_{\mathrm{CV}}$ volume size, $\mathrm{m}^{3}$

\section{Subscripts}

$\begin{array}{ll}\mathrm{i}, \mathrm{j}, \mathrm{k} & \text { scalar components } \\ \text { ini } & \text { initial } \\ \mathrm{w} & \text { wall surface } \\ \mathrm{adm} & \text { dimensionless }\end{array}$




\section{INTRODUCTION}

Compound channels are characterized by the presence of a main channel and a secondary channel, named gap. Due to geometrical and dimensional differences between channels, their velocity fields are not the same. The geometric difference and viscous flow nature promote the mass, momentum and energy transfer between the main and secondary channels. These special features give rise to a mixing layer and, under certain circumstances, large scale structures can be observed. There are innumerous shapes of compound channels. These geometries are mostly found in heat exchanger, hydraulic channels and the inner of nuclear reactors.

One of the first studies to mention the existence of flow periodic patterns in the compound channels was published by Rowe (1973), who studied the mixture process in longitudinal rod bundle under turbulent flow. Möller (1991) also performed experimental studies of this phenomenon and noticed the velocity fluctuations on the gap. In this paper the author presented a correlation for the Strouhal number as a function of the gap geometry parameters. He also presented explanation about the velocity fluctuations origins. Meyer and Rehme (1994, 1995) made measurements in compound channels using hot wire anemometry. The channels were formed by single and multslots connecting the main and secondary channels, Fig. 1. According to the authors the main dimensionless parameter was the $\mathrm{d} / \mathrm{g}$ ratio.

In the first paper the authors stated that the Strouhal number was not constant, but slightly different. The difference was produced basically due to the shape of the channel. The Strouhal numbers, formed with the main frequency, $f$, the edge velocity, $\mathrm{U}_{\text {ed }}$ and using (d.g) $)^{0.5}$ as a length scale, were found 0.066, 0.080 and 0.050, for channels from Fig. 1 (a), (b) and (c), respectively.

Studies about the flow in gaps created by tubes placed parallel to each other were developed by Krauss and Meyer (1996). The study was made over a structure of 37 parallel tubes in a symmetric horizontal hexagonal channel.

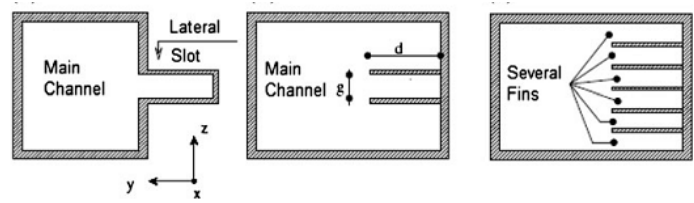

Figure 1. Compound channels studied by Meyer and Rehme. a, b) channel with single slot. c) channel with multslots.

Another experimental study about the structure of the turbulent flow surrounding cylinders in a rectangular channel was developed by Guellouz and Tavoularis (2000). Chang and Tavoularis (2007) developed a numerical study of the turbulent flow longitudinal to tubes. The physical model used was the Candu nuclear reactor, with 37 parallel tubes.

Hong et al (2011) developed numerical simulations in rectangular channels. In the center of these channels there were thin plates apart from each other by a certain distance creating a narrow gap, with distance from 5 to $15 \mathrm{~mm}$. The authors identified the intensification in the heat transfer process due to the appearance of oscillatory flow. Another paper on numerical simulation was presented by Chang and Tavoularis (2012), the simulations where developed in a rectangular channel with a tube inside, the results obtained with various turbulence models where compared.

Ultimately, the high cost and difficulties associated with the experimental techniques employment in this branch of engineering, the numerical techniques have been widely used.

In this paper a non-isothermal code was employed to evaluate the turbulent flow characteristics in a simulated compound channel. The channel contains a heated single tube inside, forming a narrow gap between the tube and bottom wall's channel. The physical model was based on the Guellouz and Tavoularis (2000) experimental work. The objective of this paper is to assess the influence of the narrow gap in the coherent structures formation, and the convective heat transfer under such conditions.

\section{PHYSIC MODEL, INITIAL AND BOUNDARY CONDITIONS}

The Figure 2 presents the cross-section of the physic model employed. The internal duct has a diameter D of $101 \mathrm{~mm}$. The dimensions were adopted to match with those of the experimental study from Guellouz and Tavoularis (2000). To ensure the completely developed flow, a length $\mathrm{L}$ of $80 \mathrm{D}$ was used.

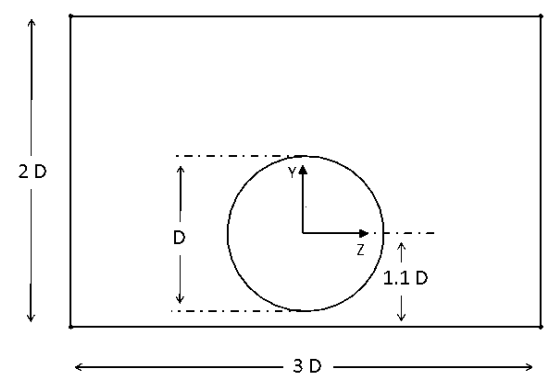

Figure 2. Cross section of physic model.

Air was adopted as working fluid and the effects of compressibility were neglected. In order to obtain a completely developed flow the problem was initially simulated considering a steady state flow, with no thermal effects. To simulate the additional diffusivity caused by the turbulence the SST turbulence model was applied. The adopted boundary conditions were: mass flow of $0.64 \mathrm{~kg} / \mathrm{s}$ and outlet 
with uniform partial pressure $\mathrm{p}=0 \mathrm{~Pa}$. Walls were considered adiabatic and no slip condition was applied.

The resulting velocity field at the exit region of this simulation was employed as inlet boundary condition to the transient simulation. In the second simulation, the buoyancy and the thermal problem were considered. Scale Adaptive Simulation (SAS) was employed as turbulence model. At this time, a thermal heat flux, q" $=2000 \mathrm{~W} / \mathrm{m}^{2}$, was prescribed on the tube's surface. In both simulations the initial condition for whole domain was $\mathrm{U}_{\text {in }}=0 \mathrm{~m} / \mathrm{s}$ and $\mathrm{T}_{\mathrm{ini}}=25^{\circ} \mathrm{C}$. For the time evolution was adopted RMS Cournt Number equal to 1 , and $5 \mathrm{~s}$ is the total simulation time.

The computational mesh employed in the simulations yielded 11230913 nods and 12039500 elements. For the mesh construction a theoretical study on the boundary layer was performed. Based on our study the first element of the mesh was placed $5.0 \times 10^{-3} \mathrm{~mm}$ away from the wall, with inflation of 14 layers and an expansion factor of 1.3 , ensuring a great number of volumes inside the boundary layer, and $\mathrm{a} \mathrm{Y}^{+}<1$.

\section{GOVERNING EQUATIONS}

To model the turbulent flow, the Boussinesq approach, generalized by kolmogorov, can be employed. With the application of the eddy viscosity concept, the average equations of mass conservation, momentum (URANS) and energy, can be written in vector notation as Eq. (1,2,3), ANSYS Inc. (2009).

$$
\begin{gathered}
\rho \frac{\partial U_{i}}{\partial t}+\frac{\partial}{\partial x_{j}}\left(\rho U_{j}\right)=0 \\
\frac{\partial\left(\rho U_{i}\right)}{\partial t}+\frac{\partial}{\partial x_{j}}\left(\rho U_{i} U_{j}\right) \\
=-\frac{\partial p^{\prime}}{\partial x_{i}}+\frac{\partial}{\partial x_{j}}\left[\mu_{e f}\left(\frac{\partial U_{i}}{\partial x_{j}}+\frac{\partial U_{j}}{\partial x_{i}}\right)\right]+S_{M} \\
\frac{\partial\left(\rho h_{t o t}\right)}{\partial t}-\frac{\partial p}{\partial t}+\frac{\partial}{\partial x_{j}}\left(\rho U_{j} h_{t o t}\right)=\frac{\partial}{\partial x_{j}}\left(\lambda \frac{\partial T}{\partial x_{j}}-\frac{\mu_{t}}{\operatorname{Pr}_{t}} \frac{\partial h}{\partial x_{j}}\right) \\
+\frac{\partial}{\partial x_{j}}\left[U_{i}\left(\tau_{i j}-\rho u_{i} u_{j}\right)\right]+S_{E} \\
-\frac{2}{3} \delta_{i j}\left(\rho k+\mu_{t} \frac{\partial U_{k}}{\partial x_{k}}\right) \\
\left(\frac{\partial U_{i}}{\partial x_{j}}+\frac{\partial U_{j}}{\partial x_{i}}\right)
\end{gathered}
$$

In the above equation $\rho$ is the density, $t$ is the time, $\mathrm{x}$ is the coordinate, $\mathrm{U}$ is the velocity vector, and the subscripts $\mathrm{i}, \mathrm{j}$ and $\mathrm{k}$ indicate the scalar components of the velocity vector, $\mathrm{p}$ represent the pressure, $\lambda$ is the thermal conductivity, $\mathrm{T}$ is the temperature, $\operatorname{Pr}_{t}$ represent the turbulent Prandtl number, $\mathrm{h}$ is the enthalpy, $\tau$ represent the molecular tensor with the normal and shear stresses components. $\rho \overline{\mathrm{u}_{\mathrm{i}} \mathrm{u}_{\mathrm{j}}}$ is the Reynolds stress tensor and represents the effects of turbulence, $S_{M}$ is the sum of the body forces, $S_{E}$ is the source term to Reynolds averaged energy conservation equation, $\mu_{t}$ is the eddy viscosity and $\delta$ is the delta of kronecker.

$$
\begin{gathered}
\mu_{e f}=\mu+\mu_{t} \\
p^{\prime}=p+\frac{2}{3}\left(\rho k+\mu_{e f} \frac{\partial U_{k}}{\partial x_{k}}\right) \\
h_{t o t}=h+\frac{1}{2} U_{i} U_{j}+k \\
k=\frac{1}{2} u_{i}^{2}
\end{gathered}
$$

Where $\mu$ is the dynamic viscosity, $\mathrm{k}$ is the turbulent kinetic energy, $\mathrm{h}_{\text {tot }}$ represent the mean total enthalpy and $u$ represent the fluctuating components.

To treat the turbulence, it was used the two equations model SST-SAS, with your default formulation implemented in the solver software ANSYS-CFX13. The equations for this formulation are shown below:

$$
\begin{aligned}
& \frac{\partial(\rho k)}{\partial t}+\frac{\partial}{\partial x_{j}}\left(\rho U_{j} k\right) \\
& =P_{k}-\rho c_{\mu} k \omega+\frac{\partial}{\partial x_{j}}\left[\left(\mu+\frac{\mu_{t}}{\sigma_{k}}\right) \frac{\partial k}{\partial x_{j}}\right] \\
& \frac{\partial(\rho \omega)}{\partial t}+\frac{\partial}{\partial x_{j}}\left(\rho U_{j} \omega\right) \\
& =\alpha \frac{\omega}{k} P_{k}-\rho \beta \omega^{2}+Q_{S A S}+\frac{\partial}{\partial x_{j}}\left[\left(\mu+\frac{\mu_{t}}{\sigma_{\omega}}\right) \frac{\partial \omega}{\partial x_{j}}\right] \\
& +\left(1-F_{1}\right) \frac{2 \rho}{\sigma_{\omega 2}} \frac{1}{\omega} \frac{\partial k}{\partial x_{j}} \frac{\partial \omega}{\partial x_{j}} \\
& \mu_{t}=\frac{\rho a_{1} k}{\max \left(a_{1} \omega, S F_{2}\right)}
\end{aligned}
$$

The SAS model, Menter and Egorov (2010), was developed to solve the turbulent flow structures inside unsteady wake regions by adding an additional term, $\mathrm{Q}_{\mathrm{SAS}}$, to the source term of the $\omega$-equation in the $\mathrm{k} \omega$-SST model. 


$$
\begin{gathered}
Q_{S A S}=\max \left[\rho \zeta_{2} \kappa S^{2}\left(\frac{L}{L_{v k}}\right)^{2}\right. \\
\left.-C \frac{2 \rho k}{\sigma_{\phi}} \max \left(\frac{1}{\omega^{2}} \frac{\partial \omega}{\partial x_{j}} \frac{\partial \omega}{\partial x_{j}}, \frac{1}{k^{2}} \frac{\partial k}{\partial x_{j}} \frac{\partial k}{\partial x_{j}}\right), 0\right] \\
L_{v k}=\max \left(\frac{\kappa S}{\left|U^{\prime \prime}\right|}, C_{S} \sqrt{\frac{\kappa \zeta_{2}}{\left(\beta / c_{\mu}\right)-\alpha}} \Omega_{C V}^{1 / 3}\right) \\
L=\frac{\sqrt{k}}{c_{\mu}^{1 / 4} \omega}, \quad \frac{S=\sqrt{2 S_{i j}} S_{i j}}{S_{i j}}=\frac{1}{2}\left(\frac{\partial U_{i}}{\partial x_{j}}+\frac{\partial U j}{\partial x_{i}}\right), \\
\left|U^{\prime \prime}\right|=\sqrt{\sum_{i}\left(\frac{\partial^{2} U_{i}}{\partial x_{j} \partial x_{j}}\right)} \mu_{t} S^{2}
\end{gathered}
$$

Where the constant have the following values: $\mathrm{c}_{\mu}=0,09, \alpha=5 / 9, \beta=0,075, \mathrm{a} 1=0,31, \zeta_{2}=5,51, \sigma_{\omega}=\sigma_{\mathrm{k}}=2$, $\sigma_{\omega 2}=1,3, \sigma_{\phi}=2 / 3, \kappa=0,41, C=2$ and $C_{S}=0,11 . \omega$ is the specific dissipation rate, $\Omega_{\mathrm{CV}}$ is the volume size, $\mathrm{L}$ is the length scale of the modeled turbulence, $\mathrm{L}_{v \mathrm{k}}$ is the Von Karman length scale, $\mathrm{P}_{\mathrm{k}}$ is the turbulence production due viscous force. $\mathrm{F} 1$ and $\mathrm{F} 2$ are the blending functions to the $\mathrm{k}-\omega$ SST models, for the more details, consult the ANSYS Inc. (2009).

\section{RESULTS}

The Figure 3 shows the inner tube external wall temperature field, normalized by the inlet temperature, $25^{\circ} \mathrm{C}$. The Figure $3 \mathrm{~A}$ is the top view of the tube, the $\mathrm{B}$ is the lateral view and Fig. $3 \mathrm{C}$ is the bottom view, where the tube is $10 \mathrm{~mm}$ distant from the wall. It is possible to notice that the temperature intensity is greater in the bottom position; it is also possible to note the oscillation in the temperature field, due to the existence of the coherent structures. The perturbation in the flow extends to the lateral, promoting oscillations in the local temperature field. In the upper part, there is also perturbation, but in smaller intensity.

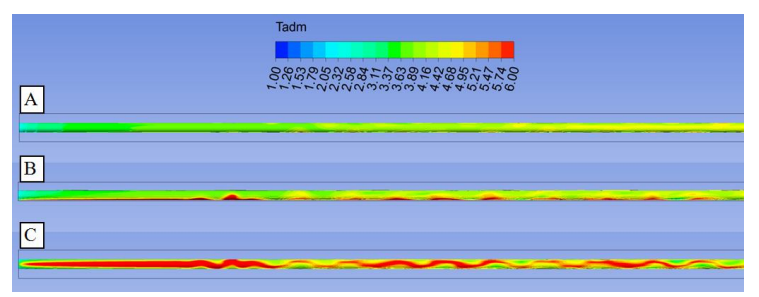

Figure 3. Temperature field in the tube wall. A) Top face. B) Lateral face. C) Bottom face.
The Figure 4A, 4B and 4C shows the temperature fields in plans placed $5 \mathrm{~mm}$ distant from the tube, in the top, lateral and bottom position. The Figure 5 show the temperature normalized in transverse plans.

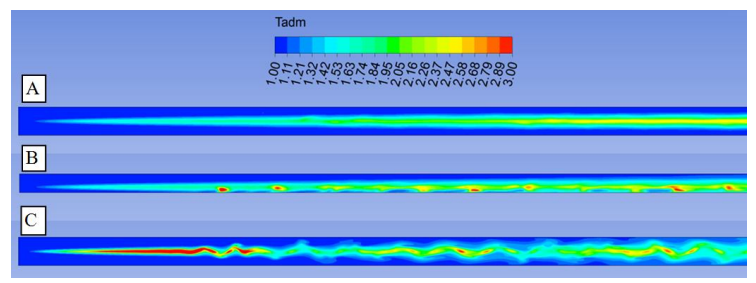

Figure 4. Temperature fields in plans $5 \mathrm{~mm}$ distant from the wall tube. A) Top plan. B) Lateral plan. C) Bottom plan.

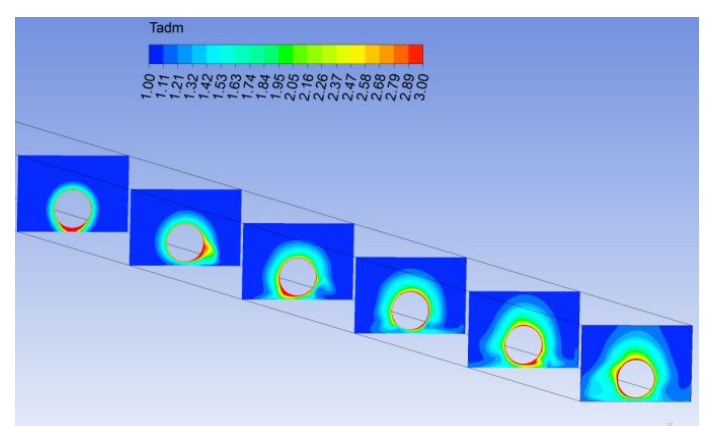

Figure 5. Temperature fields in transverse plans.

The Figure 6A, 6B and 6C show the velocity fields normalized by the velocity $\mathrm{V}_{\text {bulk }}$, in the same plans of the Fig. 4. Again, the effects of the appearing of the coherent structures can be observed.

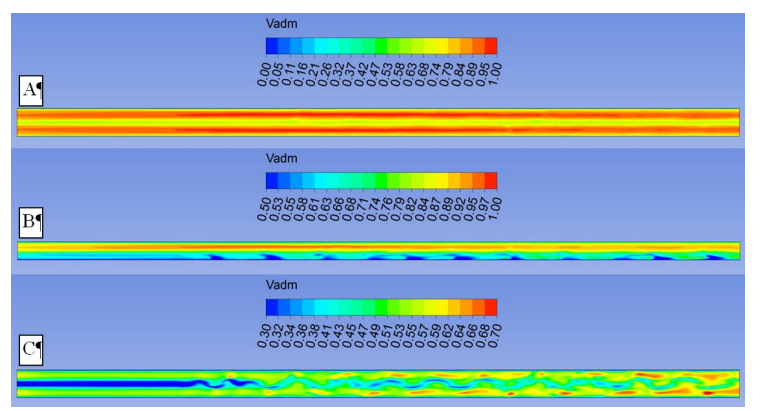

Figure 6. Instantaneous normalized velocity in plans $5 \mathrm{~mm}$ distant from the tube wall. A) Top Plane. B) Lateral plane. C) Bottom plane.

To determine the local and mean values of the convective heat transfer coefficient $h$ and the Nusselt number $\mathrm{Nu}$, Eq. 17 and 18 were used.

$$
\begin{aligned}
h & =\frac{q^{\prime \prime}}{\left(\bar{T}_{r}-\bar{T}_{f}\right)} & \bar{h} & =\frac{1}{L} \int h d x \\
N u & =\frac{h D}{\lambda} & \bar{N} u & =\frac{1}{L} \int N u d x
\end{aligned}
$$


Here $\mathrm{q}^{\prime \prime}$ is the thermal heat flux, $\overline{\mathrm{T}}_{\mathrm{r}}$ is the local mean temperature of the wall tube, $\bar{T}_{f}$ represent the local mean temperature of the fluid. The local mean temperatures were obtained in 80 plans, similar to that shown in Fig. 7, with $\Delta x$ equal to $100 \mathrm{~mm}$. The mean values where $\overline{\mathrm{h}}=23.8 \mathrm{~W} / \mathrm{m}^{2} \mathrm{~K}$ and $\overline{\mathrm{Nu}}=145$.

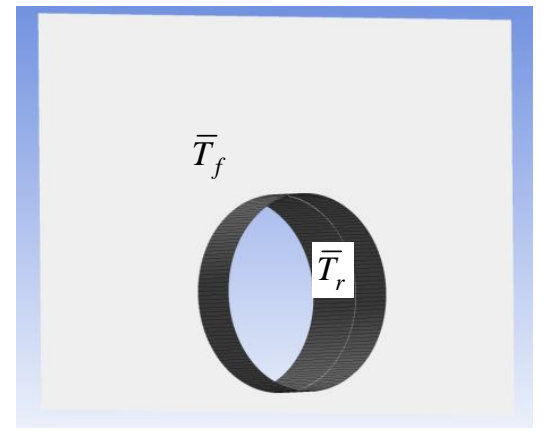

Figure 7. Plans where the mean temperatures were measured.

The Figure 8A show the behavior of transversal velocity $\mathrm{W}$ and the Fig. $8 \mathrm{~B}$ show the dimensionless temperature. The fluctuations intensity, represented by the relation $\mathrm{W} / \mathrm{V}_{\text {bulk, }}$ get at maximum values around 0.2 . These data where obtained in a line placed in the mean distance between the tube walls and the channel bottom wall. Close to X/D equal to 15 is the position where velocity fluctuations begin.

The Figure 9 shows the behavior of the heat transfer process to the X/D positions in the channel. In this figure, on the left vertical axis are the values of the local convective coefficient $h$ and in the right vertical axis are show local Nusselt number in the same positions X/D used in Fig. 8. Again we see the change in the pattern of flow near X/D position equal to 15 . Both coefficients have their maximum values at the channel inlet, where the difference between the temperature $\overline{\mathrm{T}}_{\mathrm{f}}$ and $\overline{\mathrm{T}}_{\mathrm{r}}$ are lower, after the value of this temperature difference increases causing a reduction in coefficients $h$ and $\mathrm{Nu}$, while $\mathrm{X} / \mathrm{D}$ less than 15. For $X / D$ greater than 15 , the coherent structures arise and cause the span-wise velocities fluctuations, this effect is show in Fig. 8A. The coherent structures mix the fluid, decreasing the temperature difference between the tube wall and the average temperature of the fluid in the vicinity of the tube, this is shown by the behavior of the dimensionless temperature $\mathrm{T}_{\mathrm{adm}}$, show in Fig. 8B. Analyzing the Fig. 9 is possible to realize that in the region where the transversal velocity fluctuations begin, the downward trend of the convective coefficient and the Nusselt number are interrupted. This confirms that the coherent structures interfere and alter the patterns of flow.

The most intense shear flow occurs in the region between the duct and the bottom wall of the channel and can be seen in Fig. 10 and 11. In Figure 10 two rows are placed on the Z/D equals 0.5 and -0.5 , identifying the location of the inner tube wall. In these pictures are shown the profiles of time-average velocity in transverse horizontal lines placed at half the distance between the bottom wall and the duct. The Figure 11 shows the same velocity at the positions X/D of Fig. 10, but now for the position 5 $\mathrm{mm}$ from the wall and around the tube along the angle $\theta$. The position $\theta$ equal to $0^{\circ}$ is the bottom of the tube and the position $\theta$ equal to $180^{\circ}$, is at the top of the tube.
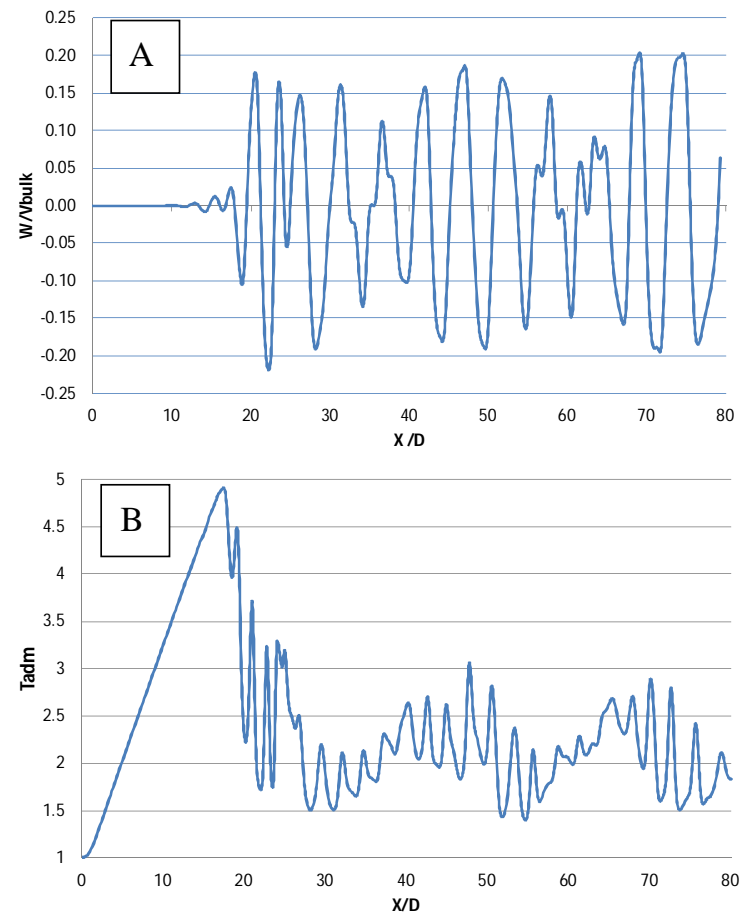

Figure 8. A) Behavior of the transversal velocity $\mathrm{W}$ (Span-Wise velocities). B) The dimensionless temperature $\left(\mathrm{T}_{\mathrm{adm}}\right)$ in the line placed in the mean distance between the tube wall and the channel bottom wall.

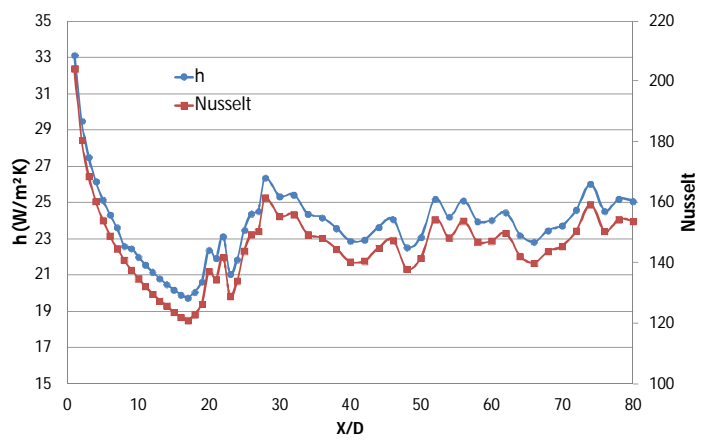

Figure 9. Local Convective Heat Transfer Coefficient and instantaneous Nusselt number.

In both figures, it is noticeable that in the gap region, the velocity gradients are more intense for Z/D less than 20. For the Z/D greater than 20, the velocity profiles are changed, decreasing the velocity gradients. This is due to the appearance of coherent 
structures in causing homogenization of momentum and thermal energy. This new shape remains until the end of the channel. In the Figure 10 also the symmetry of mean flow along the channel can be seen.

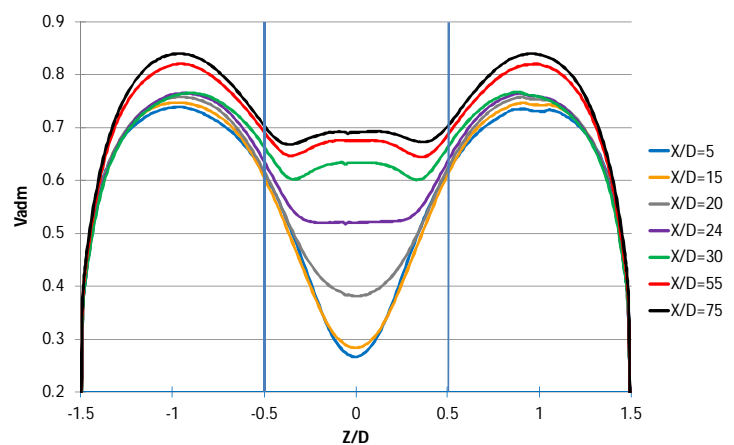

Figure 10. Normalized time-average velocity in lines placed between the tube and the bottom wall of channel.

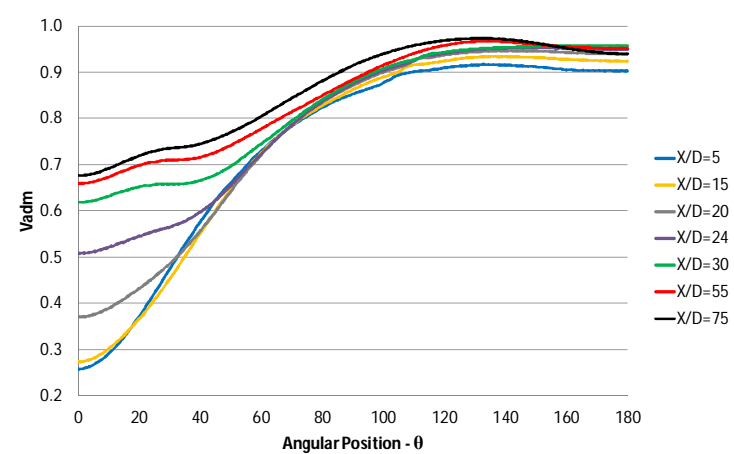

Figure 11. Normalized time-average velocity in lines around the tube.

In Figure 12 are showed the isocontours of time-average axial at X/D equal 50. On the left, the experimental results found by Guellouz and Tavoularis (2000), and in the right, the results this work. It can be observed the good agreement between the results obtained by numerical simulation and the obtained by measuring. In the simulation was found a higher value for the region where appear the greatest speeds, this difference is around 5\%, which was considered satisfactory due to physical complexity of the problem and to the difficulty modeling the turbulence.

Mean skin friction coefficient, $\mathrm{C}_{\mathrm{f}}$, was determined by the Eq. (19) and Eq. (20).

$$
\begin{gathered}
C_{f}=\frac{\tau_{w}}{\frac{1}{2} \rho V_{b u l k}^{2}} \\
V_{\text {bulk }}=\frac{1}{A} \int U_{1} d A
\end{gathered}
$$

Where $\tau_{\mathrm{w}}$ is the wall shear stress, $\mathrm{U}_{1}$ is the axial component of vector velocity, and $\mathrm{A}$ is the across section in inlet. In Figure 13 is showed both, the results obtained for this work and the experimental results obtained for Guellouz and Tavoularis (2000). These results were obtained in a position X/D equal 2.8 from the end of the channel in the bottom wall. Due to the symmetry of the average phenomenon, only one side of the channel is shown. Is possible to compare the results and again there are a good agreement between the simulation results and measurements.

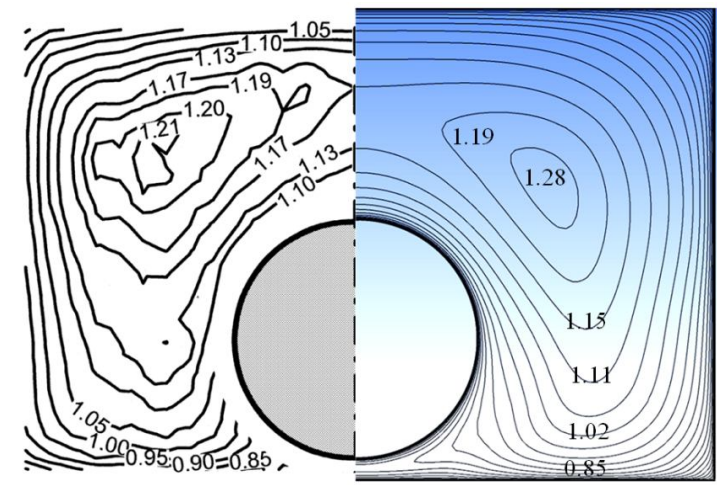

Figure 12. Isocontours of time-average axial at $\mathrm{X} / \mathrm{D}=50$, experimental results on the left Guellouz and Tavoularis, 2000, in the right side, results this work.

The

Figure 14 shows the coherent structures, identified by the vortex core region, through the channel. In this figure is possible to visualize the region of appearance of coherent structures and the region of influence of those structures on the flow in the channel surrounding the inner tube.

The large-scale structures dominating the gap, travelling at constant velocity and the Strouhal number can be formulated as follow.

$$
\begin{gathered}
S t=\frac{f D}{U_{c}} \\
f=\frac{U_{c}}{\lambda_{1}}
\end{gathered}
$$

Where $f$ is the frequency, $\mathrm{U}_{\mathrm{c}}$ is the convection velocity and the wavelength, $\lambda_{1}$, is the streamwise spacing between alternate sequence of large structures. The wavelength associated with alternate sequence of vortex can be estimated by using the average number of these structures in a certain domain, Home et al. (2009). Taking a picture of these structures and knowing the length of domain, as in Fig. 3 and 6, for example, is possible to estimate $\lambda_{1}$, as follow. 


$$
\lambda_{1}=\frac{L}{N_{\text {seq }}}
$$

Where, the length of domain is $\mathrm{L}$ and $\mathrm{N}_{\text {seq }}$, is the average number of alternate sequence of structures. For this problem Eq. (22), yields, L equal to 6350 $\mathrm{mm}$ and $\lambda_{1}$ equal to $0.453 \mathrm{~m}$.

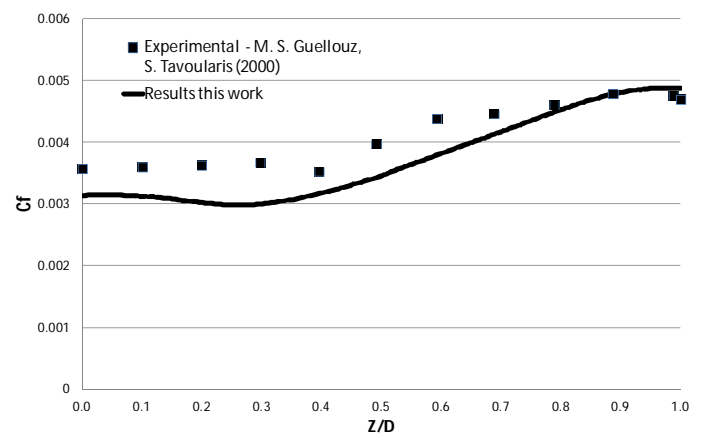

Figure 13. Mean Skin Friction Coefficient in wall under tube, $\mathrm{X} / \mathrm{D}=2.8$ from the end of the tube.

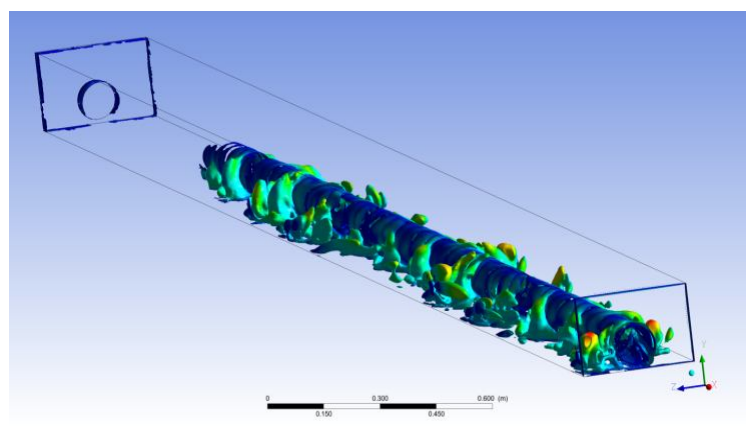

Figure 14. Vortex core region behavior near to the internal duct, isometric view by the coherent structures.

To estimate the convection velocity was used the Fig. 15. In this figure are shown the transverse velocity $\mathrm{W}$ in two positions inside the channel, positioned at half distance between the tube and the bottom wall of the channel. The first point is placed at X/D equal to 60 . The axial distance between two points was equal that $200 \mathrm{~mm}$. So, knowing the time lag between the peaks of the transverse velocity of the adjacent points, is possible estimate the convection speed, $\mathrm{U}_{\mathrm{c}}$.

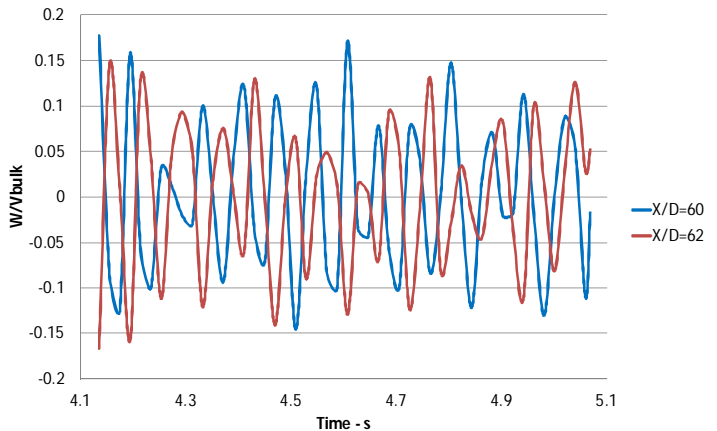

Figure 15. Behavior of the transversal dimensionless velocity $W$ in two adjacent positions.

Based on the experimental results, other authors suggested the formation of a mixed layer between main subchannel and narrow gaps. This fact may give rise to large-scale structures like similar to KelvinHelmholtz instabilities. For the flow in rectangular sub-channel geometry, the mean Strouhal numbers was reported by Meyer and Rehme (1994) as 0.115 . So, in this work, the convection velocity Uc found was around of the $4.8 \mathrm{~m} / \mathrm{s}$, the frequency $\mathrm{f}$ around $10.6 \mathrm{~s}^{-1}$ and the Strouhal number equal to 0.106 .

\section{CONCLUDING REMARKS}

In this paper turbulent flow in a channel with gap was analyzed, by using a commercial code, ANSYS-CFX13, and scale adaptive SST-SAS, as turbulence model. Analyzing the presented results, the conclusion is that the appearing of the coherent structures has an important role in the heat transfer process, due to its convection enhancement. The flow is covered by those quasi-periodic large-scale structures after the position X/D equal to 15 . These structures starts at the gap region and its influence advances to the region close to the central tube.

The Results from isocontours of time-average axial and the Mean Skin Friction Coefficient, was compared with experimental data from Guellouz and Tavoularis (2000), the results were satisfactory.

The Strouhal number obtained in this study is of the magnitude of those found in experimental studies to rectangular sub-channel geometry.

\section{REFERENCE}

ANSYS-CFX, 2009, ANSYS CFX-Solver Theory Guide, Canonsburg, PA, USA.

Chang, D., and Tavoularis, S., 2007, Numerical Simulation of Turbulent in a 37-Rod Bundle, Nuclear Engineering and Design, Vol. 237, pp. 575-590.

Chang, D., and Tavoularis, S., 2012, Numerical Simulation of Developing Flow an Vortex Street in a Rectangular Channel With a Cylindrical Core, Nuclear Engineering and Design, Vol. 243, pp. 176199.

Guellouz, M. S., and Tavoularis, S., 2000, The Structure of Turbulent Flow in a Rectangular 
Channel Containing a Cylindrical Rod - Part 1: Reynolds-Averaged Measurements, Experimental Thermal and Fluid Science, Vol. 23, pp. 59-73.

Home, D., Arvanitis, G., Linghtstone, M. F., and Hamed, M. S., 2009, Simulation of Flow Pulsation in a Twin Rectangular Subchannel Geometry using Unsteady Reynolds Average NavierStokes Modelling, Nuclear Engineering and Design, Vol. 239, 2964-2980.

Hong, S. H., Seo, J. S., Byun, J. K., Choi, Y. D., and Shin, J. K., 2011, Turbulent Heat Transfer Characteristics in Compound Channels with Gap, Journal of Mechanical Science and Technology, Vol. 25, No. 9, pp. 2217-2225.

Krauss, T., and Meyer, L., 1996, Characteristics of Turbulent Velocity and Temperature in a Wall Heated Rod Bundle, Experimental Thermal and Fluid Science, Vol. 12, pp. 75-86.

Menter F. R., and Egorov Y., 2010, The ScaleAdaptive Simulation Method for Unsteady Turbulent Flow Predictions. Part 1: Theory and Model Description, Flow Turbulence Combust, Vol. 85, pp. 113-138.

Meyer, L., and Rehme, K., 1994, Large-Scale Turbulence Phenomena in Compound Rectangular Channels, Experimental Thermal and Fluid Science, Vol. 8, pp. 286-304.

Meyer, L., and Rehme, K., 1995, Periodic Vortices in Flow Trough Channels with Longitudinal Slots or Fins, in: tenth Symposium on Turbulent Shear Flow, Pennsylvania State University, USA.

Möller, S. V., 1991, On Phenomena of Turbulent Flow Through Rod Bundles, Experimental Thermal and Fluid Science, Vol. 4, pp. 25-32.

Rowe, D. S., 1973, Measurement of Turbulent Velocity, Intensity and Scale in Rod Bundle Flow Channels, Ph.D. Thesis, Oregon State University, USA. 